\title{
Robust Adaptive Stabilization of Nonholonomic Mobile Robots with Bounded Disturbances
}

\author{
Gang Chen, ${ }^{1,2}$ Tingting Gao, ${ }^{2}$ Jiangshuai Huang, ${ }^{3}$ and Qicai Zhou ${ }^{1}$ \\ ${ }^{1}$ School of Mechanical Engineering, Tongji University, Shanghai 200092, China \\ ${ }^{2}$ School of Mechanical \& Electrical Engineering, Zhejiang Textile \& Fashion College, Ningbo, Zhejiang 315211, China \\ ${ }^{3}$ School of Electrical \& Electronic Engineering, Nanyang Technological University, Singapore 679394
}

Correspondence should be addressed to Tingting Gao; gaotingting_21@163.com

Received 6 August 2014; Accepted 24 August 2014; Published 17 November 2014

Academic Editor: Shen Yin

Copyright (C) 2014 Gang Chen et al. This is an open access article distributed under the Creative Commons Attribution License, which permits unrestricted use, distribution, and reproduction in any medium, provided the original work is properly cited.

The stabilization problem of nonholonomic mobile robots with unknown system parameters and environmental disturbances is investigated in this paper. Considering the dynamic model and the kinematic model of mobile robots, the transverse function approach is adopted to construct an additional control parameter, so that the closed-loop system is not underactuated. Then the adaptive backstepping method and the parameter projection technique are applied to design the controller to stabilize the system. At last, simulation results demonstrate the effectiveness of our proposed controller schemes.

\section{Introduction}

The motion control of nonholonomic mobile robots has been an active research field in the past few decades and remains a challenging control problem. For nonholonomic mobile robots, it cannot be stabilized by any static continuous state feedback [1], due to the Brockett's condition [2]. To handle the stability of nonholonomic systems, it has to resort to either a time-varying $[3,4]$, a discontinuous $[5,6]$, or a dynamic state feedback controller $[7,8]$.

The early work in [9] studies the tracking problem based on backstepping method for both a kinematic model and a dynamic model of mobile robot. In [10], a neural network adaptive controller based on input-output linearization design is presented to guide a mobile robot during trajectory tracking. However, these methods are only applicable when the system dynamic parameters are known. In order to handle system disturbances that are inevitable in real applications, an adaptive tracking controller has been proposed for a class of mobile robots with uncertainties [11]. Based on Lyapunov's direct method and backstepping technique [12], a time-varying global adaptive controller at the torque level that simultaneously solves both tracking and stabilization problems in the case of unknown dynamic parameters has been developed. Similar work can be found in [13]. Moreover, a robust adaptive controller is designed for a mobile robot with bounded unknown disturbances in $[14]$. The works in $[15,16]$ extend the control law design for more general uncertain nonholonomic systems. Subsequent related works on the stabilization and tracking control of nonholonomic mobile robots include, but are not limited to, [17-21] and many references therein. However, all the stabilization schemes mentioned above need the assistance of the sinusoidal function; thus, the convergence rate of stabilization is slow.

To overcome this problem, in this paper, we consider the stabilization control of the nonholonomic mobile robot with unknown parameters and bounded external disturbances. In contrast to the aforementioned results, all the system parameters as well as the disturbance are assumed to be unknown. Based on the transverse function approach [22] and backstepping techniques, a robust adaptive controller will be developed to guarantee the asymptotical stability of the nonholonomic mobile robot. By employing the traverse function approach, an "auxiliary manipulated variable" is introduced, with which the difficulty encountered in controlling an underactuated system can be overcome. The rest of this paper is arranged as follows. In Section 2, we present 


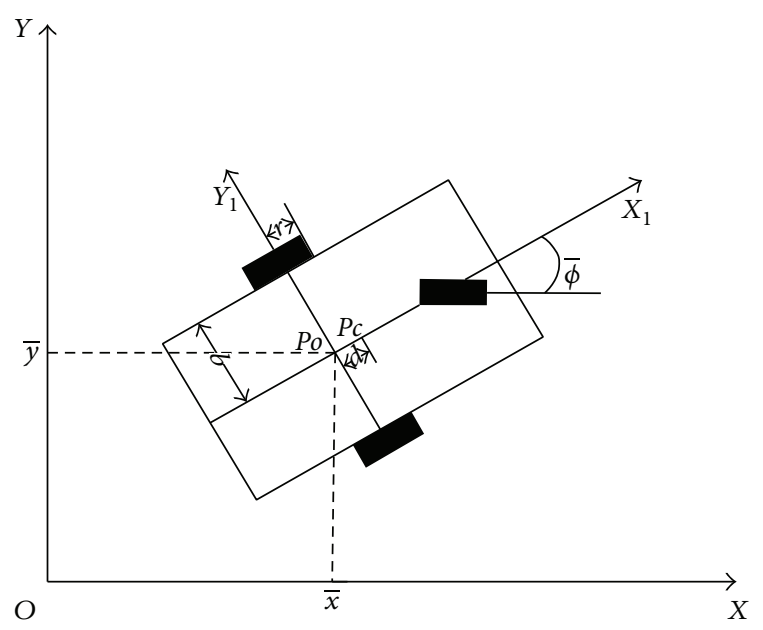

FIgURE 1: A two-wheeled nonholonomic mobile robot.

the model of nonholonomic mobile robots and problem formulation. Then we propose an adaptive control scheme to achieve stabilization in Section 3, where the stability of the overall system is discussed. Simulation results are provided in Section 4. Finally, some concluding remarks are drawn in Section 5 .

\section{Problem Formulation}

A unicycle mobile robot is considered, which consists of two driving wheels located at the same axis and a passive self-adjusted supporting wheel. The actuated two wheels are driven by two DC servomotors independently. As shown in Figure 1, the geometric center and center of mass of the mobile robot do not coincide. The origin of $P_{0}-X_{1} Y_{1}$ frame is the geometric center $P o$, the center of mass $P c$ is on $X_{1}$ axis, and the distance to the origin $P o$ is $d$. The position of $P o$ in global coordinate frame $O-X Y$ is $(\bar{x}, \bar{y})$ and $\bar{\phi}$ is the orientation of the local frame $P o-X_{1} Y_{1}$. For the sake of simplicity, it is assumed that the robot does not slip and there is no sliding between the tire and the road; that is, there is no Coulomb-like friction. Then the system can be described by the following dynamic model and kinematic model [23], where the parameters are shown in Table 1:

$$
\begin{gathered}
\dot{\eta}=J(\eta) \omega, \\
M \dot{\omega}+C(\dot{\eta}) \omega+D \omega=\tau+\tau_{d},
\end{gathered}
$$

where $\eta=(\bar{x}, \bar{y}, \bar{\phi})^{T}$ denotes the position and orientation of the robot, $\omega=\left(\omega_{1}, \omega_{2}\right)^{T}$ denotes the velocities of the left and right wheels, $\tau=\left(\tau_{1}, \tau_{2}\right)^{T}$ represents the control torques applied to the wheels, $M$ is a symmetric, positive definite inertia matrix, $C(\dot{\eta})$ is the centripetal and Coriolis matrix, $D$ denotes the surface friction, and $\tau_{d}$ is the bounded unknown
TABLE 1: Definition of parameters of mobile robot.

\begin{tabular}{ll}
\hline Symbols & Description \\
\hline$m_{c}$ & $\begin{array}{l}\text { Mass of body } \\
m_{w}\end{array}$ \\
$I_{c}$ & $\begin{array}{l}\text { Mass of wheel with a motor } \\
\text { through } P c\end{array}$ \\
$I_{w}$ & $\begin{array}{l}\text { Moment of inertia of the wheel with a motor about the } \\
\text { wheel axis }\end{array}$ \\
$I_{m}$ & $\begin{array}{l}\text { Moment of inertia of the wheel with a motor about the } \\
\text { diameter }\end{array}$ \\
$b$ & Half width of the mobile robot \\
$r$ & Radius of the wheel \\
$d_{11}, d_{22}$ & $\begin{array}{l}\text { Damping coefficients } \\
\omega_{1}, \omega_{2}\end{array}$ \\
$\tau_{1}, \tau_{2}$ & Velocities of the left and right wheels \\
$\tau_{d}$ & Control torques applied to the wheels \\
\hline
\end{tabular}

external disturbance. Matrices $J(\eta), M$, and $C(\dot{\eta})$ are the same as those in [12], which are given as

$$
\begin{gathered}
J(\eta)=\frac{r}{2}\left[\begin{array}{cc}
\cos \bar{\phi} & \cos \bar{\phi} \\
\sin \bar{\phi} & \sin \bar{\phi} \\
b^{-1} & -b^{-1}
\end{array}\right], \quad M=\left[\begin{array}{ll}
m_{11} & m_{12} \\
m_{12} & m_{11}
\end{array}\right], \\
C(\dot{\eta})=\left[\begin{array}{cc}
0 & \frac{r^{2}}{2 b} m_{c} d \dot{\bar{\phi}} \\
-\frac{r^{2}}{2 b} m_{c} d \dot{\bar{\phi}} & 0
\end{array}\right], \quad D=\left[\begin{array}{cc}
d_{11} & 0 \\
0 & d_{22}
\end{array}\right], \\
m_{11}=\frac{r^{2}}{4 b^{2}}\left(m b^{2}+I\right)+I_{w}, \quad m_{12}=\frac{r^{2}}{4 b^{2}}\left(m b^{2}-I\right), \\
m=m_{c}+2 m_{w}, \quad I=m_{c} d^{2}+2 m_{w} b^{2}+I_{c}+2 I_{m} .
\end{gathered}
$$

The upper bound of the external disturbance is assumed to satisfy

$$
\left\|\tau_{d}\right\| \leq \tau_{d \max },
$$

where $\tau_{d \max }$ is an unknown positive constant.

Two control vectors are introduced as $u_{1}=0.5\left(\omega_{1}+\omega_{2}\right)$ and $u_{2}=0.5\left(\omega_{1}-\omega_{2}\right)$, and then the kinematic model (1) can be written as

$$
\begin{gathered}
\bar{x}=r \cos \bar{\phi} u_{1}, \\
\bar{y}=r \sin \bar{\phi} u_{1}, \\
\bar{\phi}=\frac{r}{b} u_{2} .
\end{gathered}
$$

Assumption 1. The parameters $r$ and $b$ fall in known compact sets; that is, there exist some known positive constants $\bar{r}, \underline{r}, \bar{b}$, and $\underline{b}$, such that $\underline{r}<r<\bar{r}$ and $\underline{b}<b<\bar{b}$.

Remark 2. The degrees of freedom of the nonholonomic mobile robot are three, but there are only two independent control inputs, so the system (5) is underactuated. 


\section{Controller Design}

In this section, the transverse function approach is adopted firstly to perform suitable change of coordinates. With the change of coordinates, an additional controller $\dot{\xi}$ will be created and thus the kinematic model (5) is no longer underactuated. In the second step, the adaptive controller and parameter estimator are designed such that the system is stabilized.

3.1. Control Objective. Design the control inputs $\tau$ to stabilize the nonholonomic mobile robot, as modeled in (2) and (5).

3.1.1. Coordinates Transformation. The new coordinates ( $x$, $y, \phi)$ and additional controller $\xi$ are introduced, and the kinematic model (5) is transformed as follows:

$$
\begin{gathered}
{\left[\begin{array}{l}
x \\
y
\end{array}\right]=\left[\begin{array}{l}
\bar{x} \\
\bar{y}
\end{array}\right]+R(\phi)\left[\begin{array}{l}
f_{1}(\xi) \\
f_{2}(\xi)
\end{array}\right],} \\
\phi=\bar{\phi}-f_{3}(\xi)
\end{gathered}
$$

where

$$
R(\phi)=\left[\begin{array}{cc}
\cos (\phi) & -\sin (\phi) \\
\sin (\phi) & \cos (\phi)
\end{array}\right],
$$

and $f_{l}(\xi)$, for $l=1,2,3$, are functions of $\xi$ designed as

$$
\begin{aligned}
& f_{1}(\xi)=\varepsilon_{1} \sin (\xi) \frac{\sin \left(f_{3}\right)}{f_{3}}, \\
& f_{2}(\xi)=\varepsilon_{1} \sin (\xi) \frac{1-\cos \left(f_{3}\right)}{f_{3}}, \\
& f_{3}(\xi)=\varepsilon_{2} \cos (\xi),
\end{aligned}
$$

where $\varepsilon_{1}$ and $\varepsilon_{2}$ are arbitrarily small positive constants and $\varepsilon_{2}$ satisfies $0<\varepsilon_{2}<\pi / 2$. Then the following properties can be shown:

$$
\left|f_{1}\right|<\varepsilon_{1}, \quad\left|f_{2}\right|<\varepsilon_{1}, \quad\left|f_{3}\right|<\varepsilon_{2} .
$$

Taking the derivatives of $x, y$, and $\phi$ yields

$$
\begin{gathered}
{\left[\begin{array}{c}
\dot{x} \\
\dot{y}
\end{array}\right]=Q\left[\begin{array}{c}
r u_{1} \\
\dot{\xi}
\end{array}\right]+\frac{\partial R(\phi)}{\partial \phi}\left[\begin{array}{l}
f_{1}(\xi) \\
f_{2}(\xi)
\end{array}\right] \dot{\phi},} \\
\dot{\phi}=r b^{-1} u_{2}-\frac{\partial f_{3}(\xi)}{\partial \xi} \dot{\xi},
\end{gathered}
$$

where

$$
Q=\left[\left(\begin{array}{c}
\cos (\bar{\phi}) \\
\sin (\bar{\phi})
\end{array}\right) R(\phi)\left(\begin{array}{c}
\frac{\partial f_{1}(\xi)}{\partial \xi} \\
\frac{\partial f_{2}(\xi)}{\partial \xi}
\end{array}\right)\right]
$$

is ensured to be invertible [17]. Different from $(\bar{x}, \bar{y}, \bar{\phi})$, the transformed coordinates $(x, y, \phi)$ can be controlled separately by $u_{1}, u_{2}$, and $\dot{\xi}$, where $\dot{\xi}$ is an auxiliary manipulated variable to be designed; thus, (10) are not underactuated.
3.1.2. Controller Design. By replacing (5) with (10), the closedloop system composed by (2) and (10) is of strict feedback form. Therefore, the backstepping technique $[24,25]$ method is applied to design the control inputs $\tau$. Obviously, the design procedure can be divided into two steps. In the first step, the virtual controls $u_{1 d}$ and $u_{2 d}$ together with the "auxiliary manipulated variable" $\dot{\xi}$ will be constructed to stabilize the system. In the second step, the actual control signals for $\tau$ will be delivered such that $u_{1}$ and $u_{2}$ in (10) can approach the virtual controls $u_{1 d}$ and $u_{2 d}$, respectively. Apart from these, the adaptive laws for the unknown system parameters will also be provided.

Step 1. Define the parameter estimation errors $\widetilde{\theta}_{i}=\theta_{i}-\widehat{\theta}_{i}$, $i=1,2$, where $\widehat{\theta}_{1}$ and $\widehat{\theta}_{2}$ are the estimated values of $r$ and $r b^{-1}$, respectively.

Let $q=[x, y]^{T}$, and choose the Lyapunov function candidate in this step as

$$
V_{1}=\frac{1}{2} q^{2}+\frac{1}{2} \phi^{2}
$$

and then the derivative of $V_{1}$ is $\dot{V}_{1}=q^{T} \dot{q}+\phi \dot{\phi}$.

We then introduce two new error variables:

$$
u_{1 e}=u_{1}-u_{1 d}, \quad u_{2 e}=u_{2}-u_{2 d}
$$

where $u_{1 d}$ and $u_{2 d}$ are the virtual controls for $u_{1}$ and $u_{2}$, respectively. $u_{1 d}, u_{2 d}$, and $\dot{\phi}$ are chosen as

$$
\begin{gathered}
{\left[\begin{array}{c}
u_{1 d} \\
\dot{\xi}
\end{array}\right]=\left[\begin{array}{cc}
\hat{\theta}_{1}^{-1} & 0 \\
0 & 1
\end{array}\right] Q^{-1}\left(-k_{1} q-R^{\prime}(\phi)\left[\begin{array}{l}
f_{1}(\xi) \\
f_{2}(\xi)
\end{array}\right] \times\left(-k_{2} \phi\right)\right),} \\
u_{2 d}=\widehat{\theta}_{2}^{-1}\left(-k_{2} \phi+\frac{\partial f_{3}(\xi)}{\partial \xi} \dot{\xi}\right),
\end{gathered}
$$

where $k_{1}$ and $k_{2}$ are positive constants. The above design delivers the following results:

$$
\begin{gathered}
\dot{q}=-k_{1} q+Q\left[\begin{array}{c}
\tilde{\theta}_{1} u_{1}+\widehat{\theta}_{1} u_{1 e} \\
0
\end{array}\right] \\
+\frac{\partial R(\phi)}{\partial \phi}\left[\begin{array}{l}
f_{1}(\xi) \\
f_{2}(\xi)
\end{array}\right] \times\left(\widetilde{\theta}_{2} u_{2}+\widehat{\theta}_{2} u_{2 e}\right), \\
\dot{\phi}=-k_{2} \phi+\widetilde{\theta}_{2} u_{2}+\widehat{\theta}_{2} u_{2 e} .
\end{gathered}
$$

The parameter estimators for $r$ and $r b^{-1}$ are designed as

$$
\begin{aligned}
& \dot{\hat{\theta}}_{1}=\operatorname{Proj}\left(\hat{\theta}_{1}, \gamma_{\theta_{1}} \pi_{1} u_{1}\right), \\
& \dot{\hat{\theta}}_{2}=\operatorname{Proj}\left(\hat{\theta}_{2}, \gamma_{\theta_{1}} \pi_{2} u_{2}\right),
\end{aligned}
$$

where $\pi_{1}=x \cos (\bar{\phi})+y \sin (\bar{\phi}), \pi_{2}=q(\partial R(\phi) / \partial \phi)\left[\begin{array}{l}f_{1}(\xi) \\ f_{2}(\xi)\end{array}\right]+\phi$. Note that $\operatorname{Proj}(\cdot)$ denotes a Lipschitz continuous projection operator about which the design details and properties can be found in [26] and the following results are then obtained. 
Lemma 3. If $\left|\widehat{b}\left(t_{0}\right)\right| \leq b_{M}$, then the projection satisfies $\widetilde{b} \operatorname{Proj}(a, \widehat{b}) \geq \widetilde{b} a$, where $\widetilde{b}=b-\widehat{b}$.

Choose the Lyapunov function candidate in this step as

$$
V_{2}=V_{1}+\frac{1}{2 \gamma_{\theta_{1}}} \widetilde{\theta}_{1}^{2}+\frac{1}{2 \gamma_{\theta_{2}}} \widetilde{\theta}_{2}^{2}
$$

We obtain that

$$
\dot{V}_{2} \leq-k_{1} q^{T} q-k_{2} \phi^{2}+\pi_{1} \widehat{\theta}_{1} u_{1 e}+\pi_{2} \widehat{\theta}_{2} u_{2 e} .
$$

Step 2. We are at the position to derive the actual control torque $\tau$.

Define $z_{i}=\omega_{i}-\omega_{i d}, i=1,2$, where $\omega_{1 d}=u_{1 d}+$ $u_{2 d}, \omega_{2 d}=u_{1 d}-u_{2 d}$. Let $z=\left[z_{1}, z_{2}\right]^{T}$; thus, we have

$$
z=\omega-\left[\begin{array}{l}
\omega_{1 d} \\
\omega_{2 d}
\end{array}\right] .
$$

Multiplying the derivatives of both sides of (19) by $M$ and combining it with (14), we obtain that

$$
M \dot{z}=-D z+\chi^{T} \vartheta+\tau+\tau_{d},
$$

where

$$
\begin{aligned}
& \vartheta=\left[\begin{array}{lllllllll}
\frac{r^{3}}{2 b^{2}} m_{c} d & d_{11} & d_{22} & m_{11} & m_{12} & m_{11} r & m_{12} r & m_{11} r b^{-1} & m_{12} r b^{-1}
\end{array}\right], \\
& \chi=\left[\begin{array}{ccccccccc}
-\omega_{2} u_{2} & -\omega_{1 d} & 0 & -\Delta_{11} & -\Delta_{12} & -\Delta_{21} & -\Delta_{22} & -\Delta_{31} & -\Delta_{32} \\
\omega_{1} u_{2} & 0 & -\omega_{2 d} & -\Delta_{12} & -\Delta_{11} & -\Delta_{22} & -\Delta_{21} & -\Delta_{32} & -\Delta_{31}
\end{array}\right], \\
& \Delta_{1 k}=\frac{\partial \omega_{k d}}{\partial \widehat{\theta}_{1}} \dot{\hat{\theta}}_{1}+\frac{\partial \omega_{k d}}{\partial \widehat{\theta}_{2}} \dot{\hat{\theta}}_{2} \\
& \Delta_{2 k}=u_{1}\left(\frac{\partial \omega_{k d}}{\partial \bar{x}} \cos \bar{\phi}+\frac{\partial \omega_{k d}}{\partial \bar{y}} \sin (\bar{\phi})\right), \\
& \Delta_{3 k}=\frac{\partial \omega_{k d}}{\partial \bar{\phi}} u_{2}, \quad k=1,2 .
\end{aligned}
$$

Introduce the estimate $\widehat{\vartheta}$ for unknown parameter vector $\vartheta$. Then the local control torque and adaptive law are designed as

$$
\begin{gathered}
\tau=-K z-\chi^{T} \widehat{\vartheta}-0.5 \Xi-\operatorname{sgn}\left(z^{T}\right) * \widehat{\tau}_{d \max }, \\
\dot{\bar{\vartheta}}=\Gamma \chi z,
\end{gathered}
$$

where $K$ is a given positive matrix and $\widehat{\tau}_{d \max }$ is the estimate of the upper bound of $\tau_{d}, \Xi=\left[\Xi_{1}, \Xi_{2}\right]^{T}$ with

$$
\begin{aligned}
& \Xi_{1}=\pi_{1} \widehat{\theta}_{1}+\pi_{2} \widehat{\theta}_{2}, \\
& \Xi_{2}=\pi_{1} \widehat{\theta}_{1}-\pi_{2} \widehat{\theta}_{2} .
\end{aligned}
$$

The update law for $\widehat{\tau}_{d \max }$ is chosen as

$$
\dot{\hat{\tau}}_{i d \max }=\left|z_{i}\right|, \quad i=1,2 .
$$

Choose the Lyapunov function for the overall system as

$$
V_{3}=V_{2}+\frac{1}{2}\left(z^{T} M z+\widetilde{\vartheta}^{T} \Gamma^{-1} \widetilde{\vartheta}+\left\|\tau_{d}\right\|_{2}^{2}\right),
$$

where $\Gamma$ is a symmetric and positive definite matrix and $\widetilde{\vartheta}=$ $\vartheta-\widehat{\vartheta}$. We obtain that

$$
\dot{V}_{3}(t) \leq-k_{1} q_{e}^{T} q_{e}-k_{2} \phi_{e}^{2}-z^{T}(K+D) z .
$$

The main results in this section are formally presented in the following theorem.
Theorem 4. Consider the nonholonomic mobile robot system (1) and (2), with the controller (22) and parameter update laws (23) and (25) under Assumption 1. Then the closed-loop system is stable and satisfies

$$
\begin{gathered}
\lim _{t \rightarrow \infty} \bar{x}(t) \leq \sqrt{2} \varepsilon_{1}, \\
\lim _{t \rightarrow \infty} \bar{y}(t) \leq \sqrt{2} \varepsilon_{1}, \\
\lim _{t \rightarrow \infty} \bar{\phi}(t) \leq \varepsilon_{2} .
\end{gathered}
$$

Proof. Considering the projection operation, $\widetilde{\theta}_{i}, i=1,2$ are bounded. Thus from (27), all signals in $V_{3}$ are bounded. Hence, $x, y$, and $\phi$ are bounded. From (14), it is easy to check that $u_{1 d}, u_{2 d}$, and $\dot{\xi}$ are bounded. Thus $u_{1}$ and $u_{2}$ are bounded. From (26), the boundedness of $\tau$ is concluded.

From (6) and (8), we obtain that

$$
\|(x-\bar{x}, y-\bar{y})\| \leq \sqrt{2 \varepsilon_{1}^{2}}, \quad|\phi-\bar{\phi}| \leq \varepsilon_{2} .
$$

It then follows that

$$
\begin{aligned}
& |\bar{x}| \leq|\bar{x}-x|+|x|, \\
& |\bar{y}| \leq|\bar{y}-y|+|y|, \\
& |\bar{\phi}| \leq|\bar{\phi}-\phi|+|\phi| .
\end{aligned}
$$




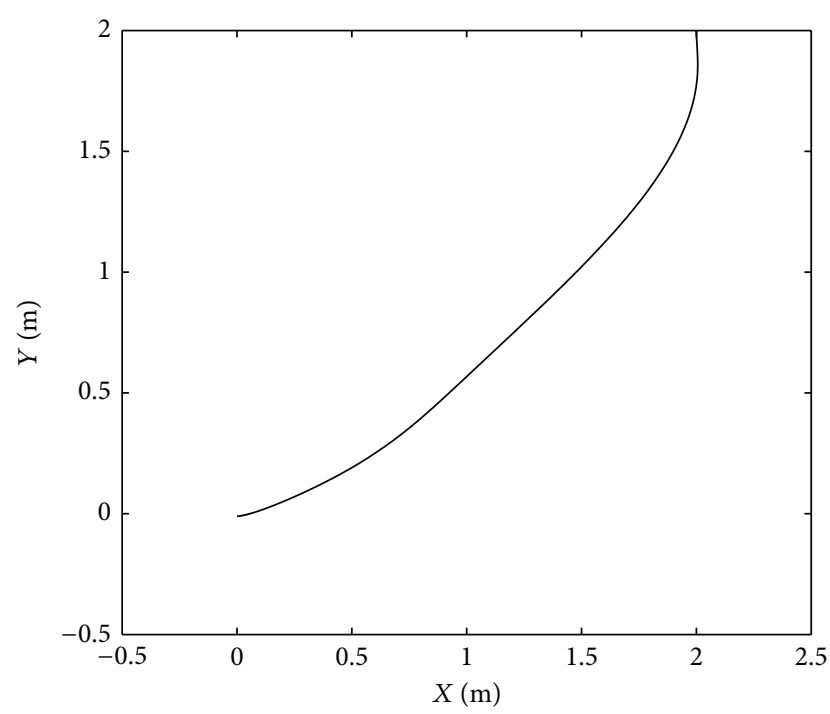

— Stabilization

FIgURE 2: The position of the robot.

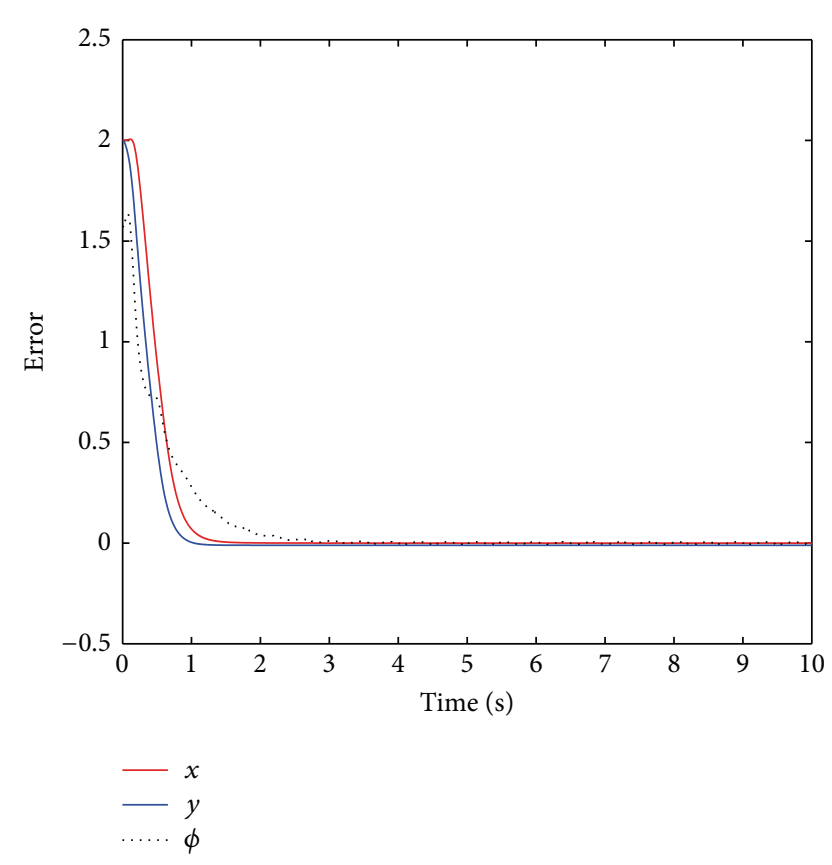

FIGURE 3: The trajectory of $x, y$, and $\phi$.

Since $x, y$, and $\phi$ will converge to zero asymptotically, (28) hold.

Remark 5. Since $\varepsilon_{1}$ and $\varepsilon_{2}$ are arbitrarily small positive constants; thus from (28) we know the stabilization errors are also arbitrarily small. The convergence rate of the system is actually close related to the design parameters $k_{1}, k_{2}$, and $D$; thus, compared to the results mentioned in the Introduction, the convergence rate can be much faster.

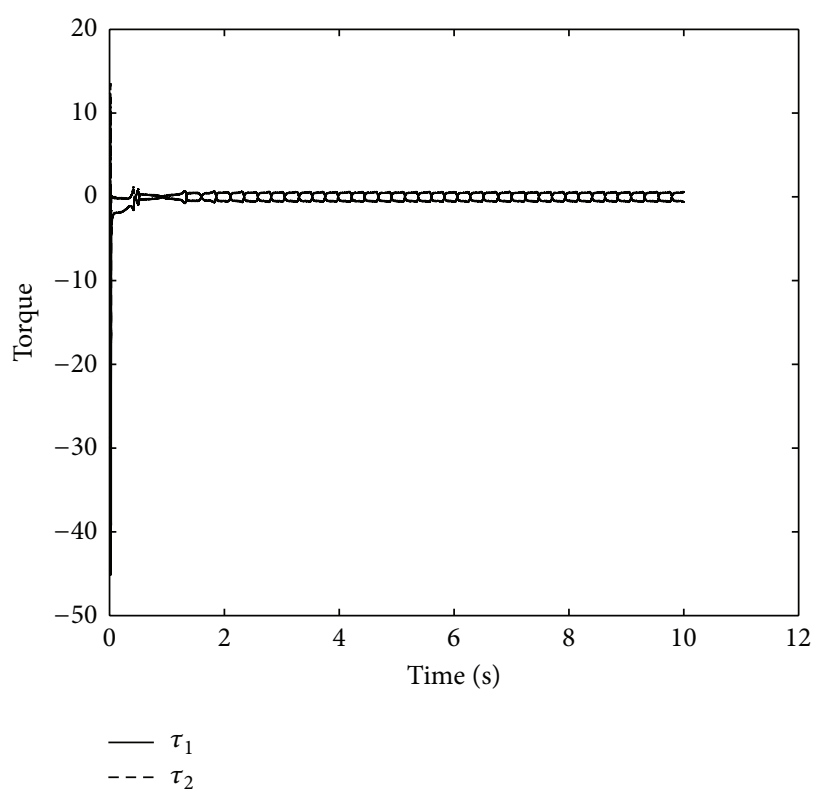

FIgURE 4: Control signals.

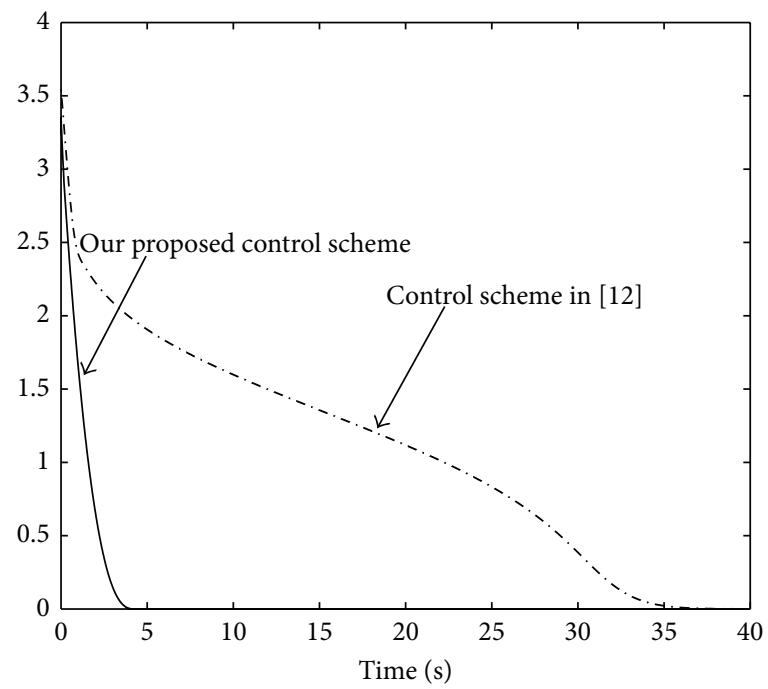

FIGURE 5: Comparison of our proposed control scheme with the scheme in [12].

TABLE 2: Simulation parameters of mobile robots.

\begin{tabular}{cc}
\hline & Parameters \\
\hline Kinematic model & $b=0.75, r=0.15$ \\
Dynamic model & $I_{c}=15.625, I_{w}=0.005, I_{m}=0.00025, m_{c}=30$, \\
& $m_{w}=1, d=0.3, d_{11}=d_{22}=5$ \\
\hline
\end{tabular}

\section{Simulation Results}

In this section, we illustrate the design procedure and how to compute the bound of the control torque based on design parameters in Table 2 using Matlab. 
The initial estimates for the unknown parameters are set to be $75 \%$ of their true values. The bounds of the external disturbances are assumed to be 10 . The design parameters are chosen as follows: $k_{1}=2, k_{2}=3, K=3 I, \varepsilon_{1}=$ $0.05, \varepsilon_{2}=0.02$. The initial conditions are set as $(2,2, \pi / 2)$. The results on the mobile robot position and tracking errors evolving with time are shown in Figures 2 and 3, respectively. It can be seen that the system stabilized in 3 seconds with the torque in Figure 4. To show that our proposed control scheme renders a faster convergence rate, we make a comparison between our control scheme and the scheme proposed in [12] in terms of $\|e\|=\sqrt{\bar{x}^{2}+\bar{y}^{2}+\bar{\phi}^{2}}$ with the same control design parameters, as shown in Figure 5. We can clearly see that the convergence rate of our proposed scheme is much faster than that of [12].

\section{Conclusions}

In this paper, stabilization problem of nonholonomic mobile robot with unknown system parameters and external disturbances is investigated. By considering the kinematic model and dynamic model of the system, the traverse function approach and the backstepping method are used to stabilize the mobile robot.

\section{Conflict of Interests}

The authors declare that there is no conflict of interests regarding the publication of this paper.

\section{References}

[1] D. Zhang, C. Wang, H. Chen, F. Yang, and J. Du, "Adaptive stabilization of stochastic non-holonomic systems with nonhomogeneous uncertainties," Transactions of the Institute of Measurement and Control, vol. 35, no. 5, pp. 648-663, 2013.

[2] R. W. Brockett, "Asymptotic stability and feedback stabilization," in Differential Geometric Control Theory, vol. 27, pp. 181-191, Birkhäuser, Boston, Mass, USA, 1983.

[3] T. C. Lee, "Exponential stabilization for nonlinear systems with applications to nonholonomic systems," Automatica, vol. 39, no. 6, pp. 1045-1051, 2003.

[4] Z. Sun, S. S. Ge, W. Huo, and T. H. Lee, "Stabilization of nonholonomic chained systems via nonregular feedback linearization," Systems \& Control Letters, vol. 44, no. 4, pp. 279289, 2001.

[5] C. Samson, "Control of chained systems application to path following and time-varying point-stabilization of mobile robots," IEEE Transactions on Automatic Control, vol. 40, no. 1, pp. 6477, 1995.

[6] J. Hespanha and A. Morse, "Stabilization of nonholonomic integrators via logic-based switching," Automatica, vol. 35, no. 3, pp. 385-393, 1999.

[7] I. Kolmanovsky and N. H. McClamroch, "Developments in nonholonomic control problems," IEEE Control Systems Magazine, vol. 15, no. 6, pp. 20-36, 1995.

[8] O. J. Sordalen and O. Egeland, "Exponential stabilization of nonholonomic chained systems," IEEE Transactions on Automatic Control, vol. 40, no. 1, pp. 35-49, 1995.
[9] Z. P. Jiang and H. Nijmeijer, "Tracking control of mobile robots: a case study in backstepping," Automatica, vol. 33, no. 7, pp. 1393-1399, 1997.

[10] C. Raimundez and A. Blas, "Adaptive tracking in mobile robots with input-output linearization," in Proceedings of the 39th Annual Conference of the IEEE Industrial Electronics Society (IECON '13), pp. 3299-3304, Vienna, Austria, 2013.

[11] T. Fukao, H. Nakagawa, and N. Adachi, "Adaptive tracking control of a nonholonomic mobile robot," IEEE Transactions on Robotics and Automation, vol. 16, no. 5, pp. 609-615, 2000.

[12] K. D. Do, Z. P. Jiang, and J. Pan, "Simultaneous tracking and stabilization of mobile robots: an adaptive approach," IEEE Transactions on Automatic Control, vol. 49, no. 7, pp. 1147-1152, 2004.

[13] F. Pourboghrat and M. P. Karlsson, "Adaptive control of dynamic mobile robots with nonholonomic constraints," Computers and Electrical Engineering, vol. 28, no. 4, pp. 241-253, 2002.

[14] R. Fierro and F. L. Lewis, "Control of a nonholonomic mobile robot: Backsteppins kinematics into dynamics," Journal of Robotic Systems, vol. 14, no. 3, pp. 149-163, 1997.

[15] Z. Jiang, "Robust exponential regulation of nonholonomic systems with uncertainties," Automatica, vol. 36, no. 2, pp. 189209, 2000.

[16] Y. Hu, S. S. Ge, and C. Su, "Stabilization of uncertain nonholonomic systems via time-varying sliding mode control," IEEE Transactions on Automatic Control, vol. 49, no. 5, pp. 757-763, 2004.

[17] Y. Shen, S. X. Ding, X. C. Xie, and H. Luo, "A review on basic data-driven approaches for industrial process monitoring," IEEE Transactions on Industrial Electronics, vol. 61, no. 11, pp. 6418-6428, 2014.

[18] Y. Shen, X. Li, H. Gao, and O. Kaynak, "Data-based techniques focused on modern industry: an overview," IEEE Transactions on Industrial Electronics, 2014.

[19] Y. Shen, W. Guang, and Y. Xu, "Robust PLS approach for KPIrelated prediction and diagnosis against outliers and missing data," International Journal of Systems Science, vol. 45, no. 7, pp. 1375-1382, 2014.

[20] Y. Shen, X. P. Zhu, and H. R. Karimi, "Quality evaluation based on multivariate statistical methods," Mathematical Problems in Engineering, vol. 2013, Article ID 639652, 10 pages, 2013.

[21] Y. Shen, X. Gao, H. R. Karimi, and X. P. Zhu, "Study on support vector machine-based fault detection in tennessee eastman process," Abstract and Applied Analysis, vol. 2014, Article ID 836895, 8 pages, 2014.

[22] P. Morin and C. Samson, "Practical stabilization of driftless systems on Lie groups: the transverse function approach," IEEE Transactions on Automatic Control, vol. 48, no. 9, pp. 1496-1508, 2003.

[23] N. Sarkar, X. Yun, and V. Kumar, "Control of mechanical systems with rolling constraints. Application to dynamic control of mobile robots," International Journal of Robotics Research, vol. 13, no. 1, pp. 55-69, 1994.

[24] J. Huang, C. Wen, W. Wang, and Z. Jiang, "Adaptive stabilization and tracking control of a nonholonomic mobile robot with input saturation and disturbance," Systems \& Control Letters, vol. 62 , no. 3, pp. 234-241, 2013. 
[25] K. D. Do and J. Pan, "Nonlinear formation control of unicycletype mobile robots," Robotics and Autonomous Systems, vol. 55, no. 3, pp. 191-204, 2007.

[26] M. Krstic, I. Kanellakopoulos, and P. V. Kokotovic, Nonlinear and Adaptive Control Design, Wiley, New York, NY, USA, 1995. 


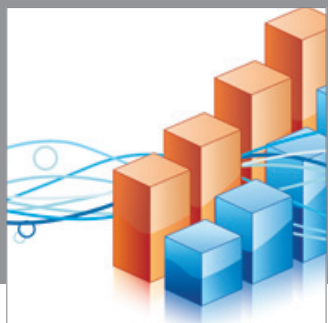

Advances in

Operations Research

mansans

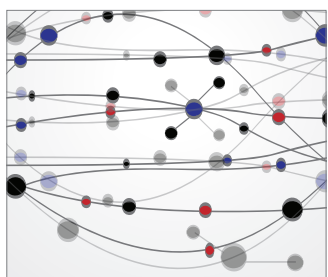

The Scientific World Journal
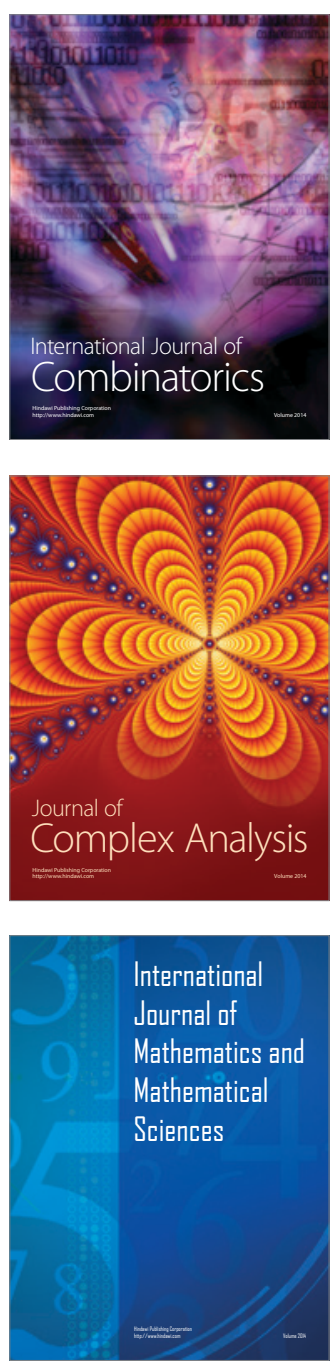
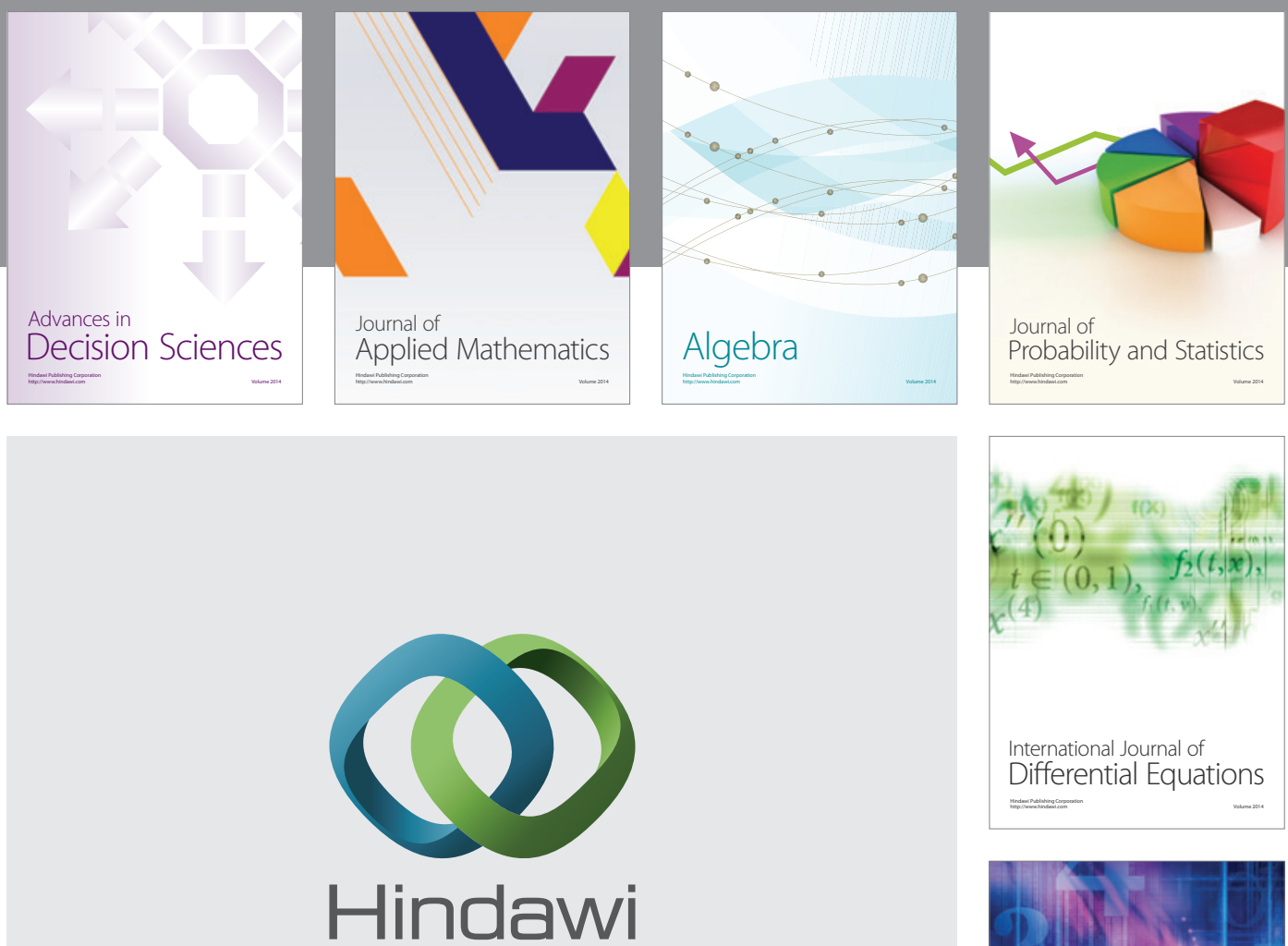

Submit your manuscripts at http://www.hindawi.com
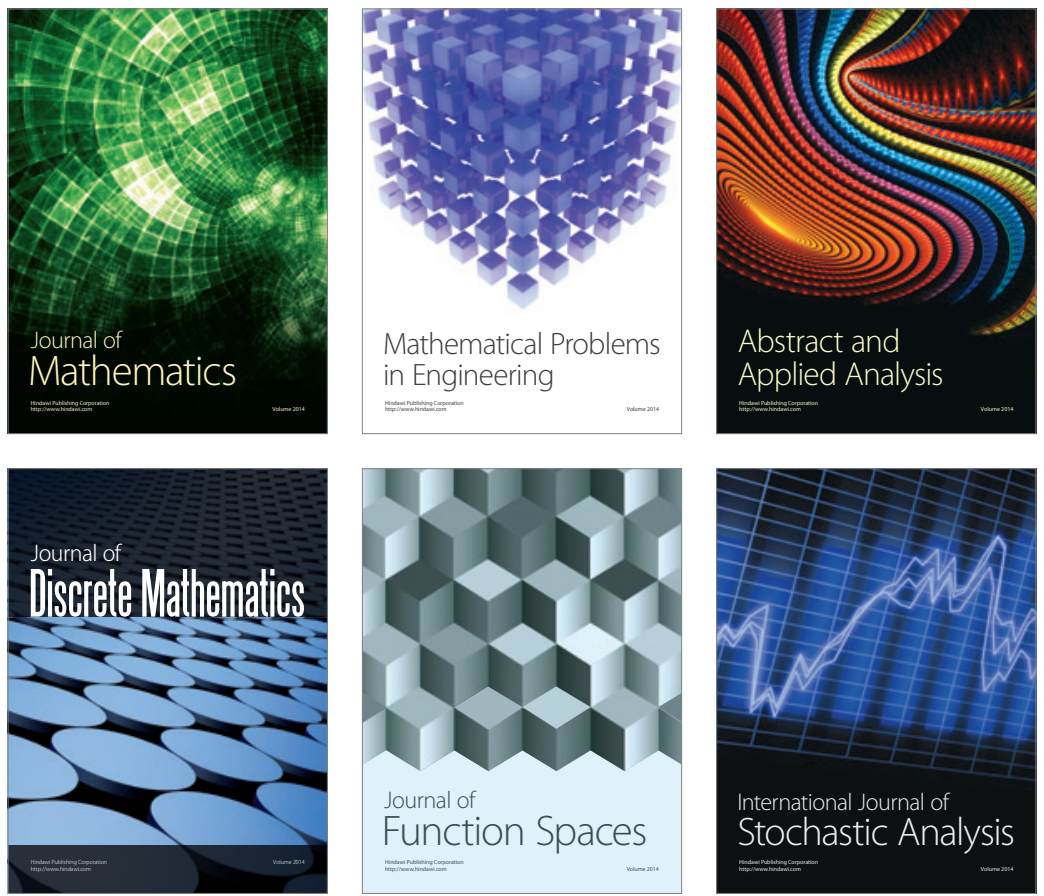

Journal of

Function Spaces

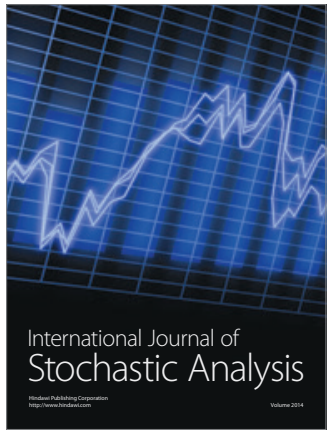

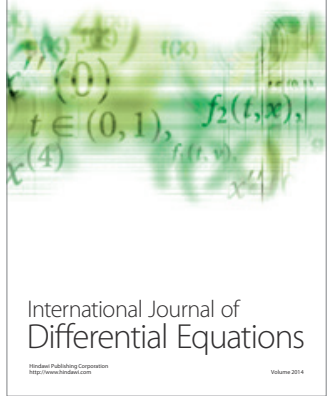
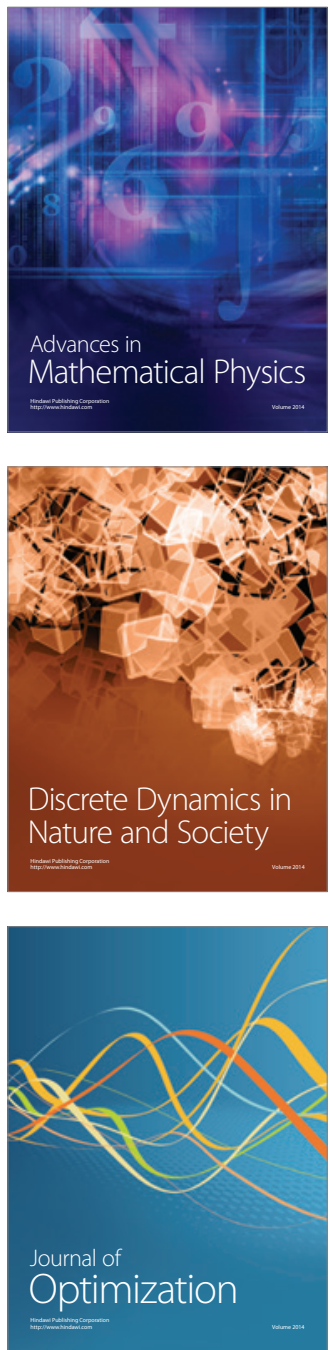